\title{
Losses Approximation for Soft Magnetic Composites Based on a Homogenized Equivalent Conductivity
}

\author{
Xiaotao Ren $^{1 *}$, Romain Corcolle $^{1,2}$, and Laurent Daniel ${ }^{1}$ \\ ${ }^{1}$ GeePs |Group of electrical engineering-Paris, UMR CNRS 8507, CentraleSupélec, Univ. Paris-Sud, \\ Université Paris-Saclay, Sorbonne Universités, UPMC Univ Paris 06 \\ 3 \& 11 rue Joliot-Curie, Plateau de Moulon 91192 Gif-sur-Yvette CEDEX, France \\ ${ }^{2}$ NYU Shanghai, Shanghai, China \\ *corresponding author, E-mail: xiaotao.ren@geeps. centralesupelec.fr
}

\begin{abstract}
Soft magnetic composites (SMC) can be used as a replacement material for laminated steel in many Electrical Engineering applications since they offer low levels of eddy current (EC) losses. However, appropriate tools are required to determine the electromagnetic behavior of SMC. Standard numerical techniques such as the finite element method can indeed lead to high computational cost to incorporate the material microstructure. Another difficulty lies in the high property contrast between the matrix and the inclusions. In this paper we propose a homogenization strategy to define the equivalent electromagnetic properties of SMC. The strategy relies on the determination of an equivalent conductivity and permeability for the material. These equivalent properties can be used to calculate eddy current losses or introduced into structural analysis tools to design electromagnetic devices.
\end{abstract}

\section{Introduction}

SMC consists in ferromagnetic inclusions embedded in a dielectric polymer matrix. This microstructure explains the low level of EC losses observed in these materials when they are subjected to electromagnetic field. This is why they are considered as a promising alternative to laminated steel, for instance in motors $[1,2,3,4]$.

The optimization of material properties is key to the design of electrical machines using SMC. In order to reach high effective magnetic permeability, pure iron or Fe-alloys are often used as the particle material [5]. In order to limit EC losses, epoxy is usually chosen as the dielectric coating. These constituents exhibit a very high conductivity and permeability contrast. In order to properly design electromagnetic devices using SMC, accurate modelling tools are required. The typical working frequency range for SMC used in AC magnetic fields is usually below $10 \mathrm{kHz}$.

Finite Element Method (FEM) is a full-field approach to solve electromagnetic problems, and provides detailed information about the field distribution all over the study domain. Effective permeability and EC losses can be readily obtained from such an approach. If the microstructure is complex, as in the case of SMC, efficient meshing processes are required and the resolution procedures can bring significant numerical complexity and instabilities. Numerical homogenization strategies derived from standard FEM have been proposed $[6,7]$ to reduce the computational time and resources to a certain extent while maintaining accuracy. However, the flexibility of these techniques for instance for parametric studies is still limited since it requires multiple numerical computations.

Other homogenization techniques have been introduced to tackle electromagnetic problems with different scales, mainly by replacing composite periodical microstructures by an equivalent homogeneous material $[8,9,10]$. From this perspective, homogenization can be particularly suitable for SMC. The equivalent homogeneous material can be used in standard structural analysis tools, with a reduced numerical complexity.

Mean field analytical or semi-analytical approaches are another homogenization strategy. These techniques have for instance been developed for the determination of the effective magnetic permeability of ferromagnetic polycrystals $[11,12]$. They have also been used for the effective permittivity of composites for shielding applications [13, 14, 15]. Préault et al. have developed a Dynamic Homogenization Method (DHM) to extend homogenization models to a wider frequency range by introducing the ratio between the typical size of the microstructure and the typical length of the electromagnetic wave [15]. Nevertheless, DHM, being an extension of Maxwell-Garnett (MG) model, is restricted to composites with low property contrast. In this paper, an analytical homogenization strategy is proposed to estimate the EC losses in SMC under standard operating conditions for electrical machines by defining an equivalent conductivity based on a loss equivalence.

In a first part, homogenization principles are briefly presented and the specificities of their application to SMC are explained. In a second part, a strategy to handle these specificities is detailed, defining an equivalent conductivity based on an equivalence in EC losses. An example of the homogenization implementation on complex components is given and the validity frequency range is finally discussed. 


\section{Homogenization of losses in SMC}

Homogenization can be a useful tool when the numerical study of a device involves different scales, which would lead to non-manageable numerical systems. The principle of homogenization is to replace a heterogeneous material with a fictitious equivalent homogeneous medium (EHM) exhibiting the same macroscopic behavior. For SMC, the parameters of interest are the effective magnetic permeability and the level of EC losses.

Since this study is limited to frequencies below $10 \mathrm{kHz}$ and linear properties, the effective magnetic permeability can be deduced from classical homogenization approaches (Wiener or Maxwell-Garnett estimates), the magnetic field induced by eddy currents being negligible. For a composite made of ferromagnetic inclusions with permeability $\mu_{2}$ embedded in a matrix with permeability $\mu_{1}$, the effective anisotropic permeability (diagonal tensor) of the mixture is

$$
\tilde{\mu}_{i}=\mu_{1}+\frac{v_{2} \mu_{1}\left(\mu_{2}-\mu_{1}\right)}{\mu_{1}+\left(1-v_{1}\right) N_{i}\left(\mu_{2}-\mu_{1}\right)}, i=x, y, z
$$

where $v_{2}$ is the volume fraction of the inclusions, $N_{i}$ is the depolarization factor in the $i$ direction [16] .

However, the approach is not applicable to the effective electric conductivity because of the very different current distribution in the heterogeneous and homogeneous cases. To obtain a relevant loss estimate, an equivalent conductivity approach is defined, based on a total EC losses equivalence.

\subsection{EC losses in SMC}

The EC losses density $\mathcal{U}\left(\mathrm{J} / \mathrm{m}^{3}\right)$ are defined as the Joule losses dissipated per unit volume during a wave period:

$$
\mathcal{U}=\frac{\left\langle\mathbf{E}^{*} \overline{\bar{\sigma}} \mathbf{E}\right\rangle}{2 f}
$$

where $f$ is the frequency of the electric field $\mathbf{E}$ within the considered domain and $\overline{\bar{\sigma}}$ is the electric conductivity tensor. The operator $\langle\cdot\rangle$ denotes a volume average over the domain and the superscript $*$ symbol refers to conjugate transpose.

In addition to the geometry, EC losses in SMC are intricately connected to the material microstructure. A model of losses in SMC needs then to incorporate a description of the microstructure. The microstructure is approximated here as a periodic pattern of ferromagnetic grains embedded in a dielectric domain. Fig. 1 gives a representation of a plate structure made of SMC with this microstructure and of an elementary cell consisting of a grain and its surrounding matrix. All numerical applications have been performed considering Iron (conductivity $\sigma_{2}=1.12 \times 10^{7}$ $\mathrm{S} / \mathrm{m}$, permeability $\left.\mu_{2}=4000 \mu_{0}\right)$ as the inclusion material and Epoxy $\left(\sigma_{1}=1.7 \times 10^{-13} \mathrm{~S} / \mathrm{m}, \mu_{1}=\mu_{0}\right)$ for the matrix.

A time-harmonic magnetic flux is imposed perpendicularly to the domain ( $z$ direction). EC can be separated into a part contained inside the ferromagnetic inclusions (in-grain current linked to the microstructure) and another

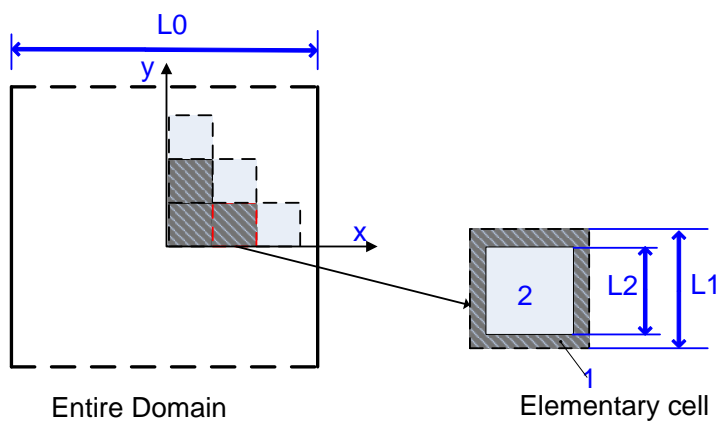

Figure 1: Periodic microstructure of composite with two materials 1 (matrix) and 2 (ferromagnetic grains).

part flowing from grain to grain through the matrix (global current linked to both microstructure and structure). For the typical working conditions where the conductivity contrast between grains and matrix is high and the exciting frequency low, the second contribution is negligible, as shown in Fig. 2.
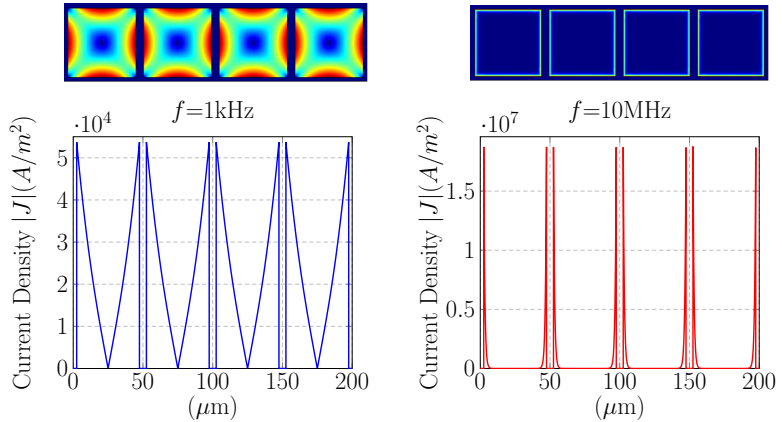

Figure 2: EC distributions in a SMC microstructure at $1 \mathrm{kHz}$ and $10 \mathrm{MHz}$ (horizontal center line).

The global losses are then limited to the sum of the losses in all independent grains. For the microstructure considered here, EC losses in each elementary cell can be calculated analytically.

\subsection{Problematics with homogenization of SMC}

Contrary to EC in the heterogeneous SMC, the induced EC appearing in a homogeneous material is only a global current. The distribution of this global current is linked to the structure (geometry) of the material, as shown in Fig. 3.

The EC distributions are totally different in the heterogeneous and homogeneous cases and cannot be readily deduced one from the other. However an equivalence on the total EC losses can be considered to define an effective electric conductivity.

\section{Definition of the equivalent conductivity}

In order to homogenize SMC, a particular definition of the equivalent conductivity can be used in order to link the losses between the composite and the corresponding ho- 


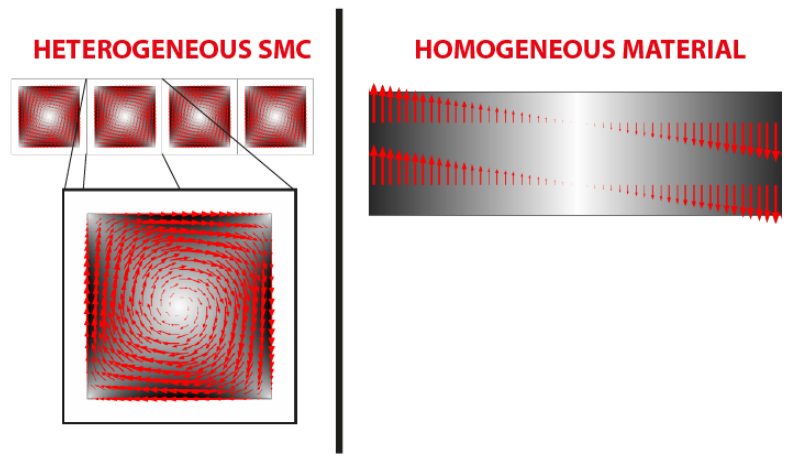

Figure 3: EC distribution in heterogeneous and homogeneous material for the plate presented in Fig. 1.

mogenized material, even though they have completely different EC distributions.

The homogenization strategy to determine equivalent conductivity is indicated in Fig. 4.

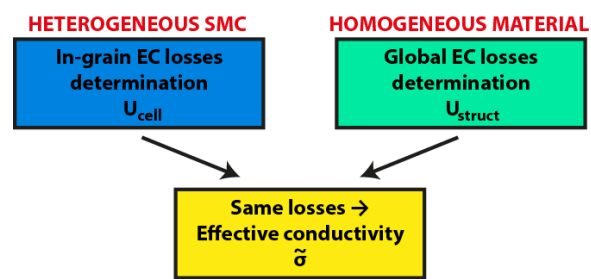

Figure 4: Homogenization strategy to keep consistency for the evaluation of losses.

EC losses of the composite equals that of a cell. Then an equivalent conductivity deduced from $\mathcal{U}_{\text {struct }}=\mathcal{U}_{\text {cell }}$ can represent the conductivity of the composite from the point of view of losses.

\subsection{EC losses in the homogeneous structure}

For laminated materials, the EC losses are proportional to $f \sigma B^{2}[17,18]$. A detailed derivation can be found in [19]. From analytical deductions on simple shapes and substantial numerical simulations on complex structures, we find that, at low frequency range for arbitrary 2D structure, the losses in a homogeneous material subjected to a magnetic flux normal to the domain are always proportional to the conductivity $\sigma$, frequency $f$, and to the square of magnetic field magnitude $B$ and typical size $L$ :

$$
\mathcal{U}=K f \sigma B^{2} L^{2}
$$

where $K$ is a shape coefficient concerning only the geometry.

For simple shapes such as a circle, a square and a plate, with the low frequency assumption, the EC losses can be mathematically approximated[19, 20],

$$
\mathcal{U}=\left\{\begin{array}{cl}
\frac{9 \pi^{2}}{128} f \sigma B^{2} L^{2} & \text { for a square (side } L \text { ) } \\
\frac{\pi^{2}}{6} f \sigma B^{2} L^{2} & \text { for a plate (width } L \text { ) } \\
\frac{\pi^{2}}{4} f \sigma B^{2} R^{2} & \text { for a circle (radius } R \text { ) }
\end{array}\right.
$$

where the formulas for circle and plate are exact but the one for square is estimated from numerical calculations.

\subsection{EC losses in the cell}

In the case presented in Fig. 1, an elementary cell is made of two materials with different conductivities. Since EC only appears in the inclusion, the determination of the losses in the material reduces to the calculation of the losses in the arbitrary-shaped grain of material 2 :

$$
\mathcal{U}_{\text {cell }}=v_{2} \mathcal{U}_{2}=v_{2} \frac{\left\langle\mathbf{E}^{*} \overline{\bar{\sigma}} \mathbf{E}\right\rangle_{2}}{2 f}
$$

where $v_{2}$ is the volume fraction of inclusion. The operator $\langle\cdot\rangle_{2}$ denotes a volume average over the grain. Based on (3) and (4), the expression of EC losses in a cell can be simplified into:

$$
\mathcal{U}_{\text {cell }}= \begin{cases}v_{2} \frac{9 \pi^{2}}{128} f \sigma_{2} B_{2}^{2} L_{2}^{2} & \text { square (side } \left.L_{2}\right) \\ v_{2} \frac{\pi^{2}}{4} f \sigma_{2} B_{2}^{2} R^{2} & \text { circular (radius } R \text { ) }\end{cases}
$$

where $B_{2}$ is the magnitude of the magnetic induction in the grain $\left(B_{2}=B \cdot \mu_{2} / \tilde{\mu}_{z}\right.$ with $B$ the average magnetic induction and $\tilde{\mu}_{z}$ the Wiener effective permeability [12]). For other shapes of inclusion, a FEM calculation on the cell is needed to determine the shape coefficient for the microstructure.

\subsection{Equivalent Conductivity}

A real component made of SMC is homogenized to be replaced by a material of equivalent conductivity $\hat{\sigma}$.

For example, as for a real square-shaped component with the side length $L$, letting $L=N L_{1}$, made of SMC configured as Fig. 1, the equivalent conductivity $\hat{\sigma}$ is obtained by equalizing (4) and (6). This leads to the definition of equivalent conductivity:

$$
\hat{\sigma}=v_{2} \sigma_{2}\left(\frac{\mu_{2}}{\tilde{\mu}_{z}}\right)^{2}\left(\frac{L_{2}}{L}\right)^{2}=v_{2}^{3} \sigma_{2} \frac{1}{N^{2}}\left(\frac{\mu_{2}}{\tilde{\mu}_{z}}\right)^{2}
$$

This equation indicates that for a given component, the smaller this cell is (i.e. a bigger $N$ ), the smaller equivalent conductivity should be, which would lead to less EC losses. It is consistent with classical observation for SMC.

For more complex geometries, a FEM computation with homogeneous conductivity is necessary to identify the proportionality coefficient for $\mathcal{U}$. Generally, the inclusion is of simple shape such as square or circle, where the shape coefficient $K$ is one of (4). The equivalent conductivity can then be determined.

\subsection{Practical Implementation}

The homogenization model is applied to three different configurations :

(a) a circular component with square inclusions,

(b) a plate composed of ellipsoidal inclusions, 
(c) a hexagonal component with circular inclusions.

The geometry is given in Fig. 5

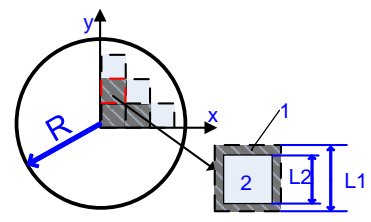

(a)

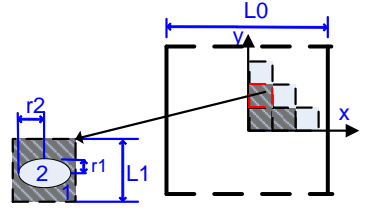

(b)

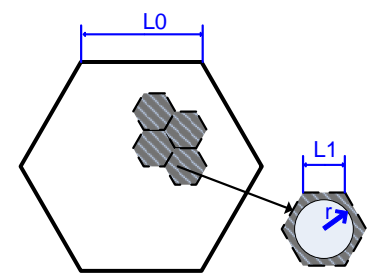

(c)

Figure 5: Three configurations components of SMC to implement homogenization model.

For case (a), both the $\mathcal{U}_{\text {struct }}$ and $\mathcal{U}_{\text {cell }}$ can be readily obtained analytically from (4) and (6). The equivalent conductivity is

$$
\hat{\sigma}_{a}=\frac{9}{32} v_{2} \sigma_{2}\left(\frac{\mu_{2} L_{2}}{\tilde{\mu} R}\right)^{2}
$$

For case (b), $\mathcal{U}_{\text {struct }}$ can be selected from (4), while $\mathcal{U}_{\text {cell }}$ should be calculated from FEM. Set $r_{1}=20 \mu \mathrm{m}$ and $r_{2}=24 \mu \mathrm{m}$ and do FEM on this homogeneous ellipse, it is easy to retrieve that the shape coefficient $K_{\text {cell }}=2.4167$. Since

$$
\mathcal{U}_{\text {cell }}=v_{2} K_{\text {cell }} f \sigma_{2} B_{2}^{2} r_{1} r_{2},
$$

now we can deduce the equivalent conductivity for this configuration,

$$
\hat{\sigma}_{b}=\frac{6 r_{1} r_{2} \mu_{2}^{2}}{\pi^{2} L_{0}^{2} \tilde{\mu}^{2}} v_{2} K_{\text {cell }} \sigma_{2}
$$

where $r_{1} r_{2}$ represents the size square stated in (3).

For case (c), $\mathcal{U}_{\text {struct }}$ has to be computed numerically. Given $L_{0}=2050 \mu \mathrm{m}$, the corresponding $K_{c}$ is $K_{c}=$ 1.975. The equivalent conductivity is

$$
\hat{\sigma}_{c}=\frac{\pi^{2}}{4 K_{c}} v_{2} \sigma_{2}\left(\frac{\mu_{2} r}{\tilde{\mu} L_{0}}\right)^{2}
$$

Set structure length to be about $2 \mathrm{~mm}$ ( $R=$ $2005 \mu \mathrm{m}, L_{0}^{b}=2000 \mu \mathrm{m}$ and $L_{0}^{c}=2050 \mu \mathrm{m}$ ), and cell length at $50 \mu \mathrm{m}$. EC losses as a function of frequency, obtained from a full FEM performed on the heterogeneous structure - for validation purpose - and from the homogenized model are indicated in Fig. 6. The two plots indicate that at low frequency (here less than $10 \mathrm{kHz}$ ) the homogenization model provides accurate losses estimate, with errors less than 1\%. At high frequency such as $\mathrm{f}=10 \mathrm{kHz}$, the homogenization model does not apply. Besides, for the hexagon case, the size of the inclusion $42 \mu \mathrm{m}$ is bigger than the other two cases, so that the valid frequency range is more narrow, as will be demonstrated in the following discussion section.
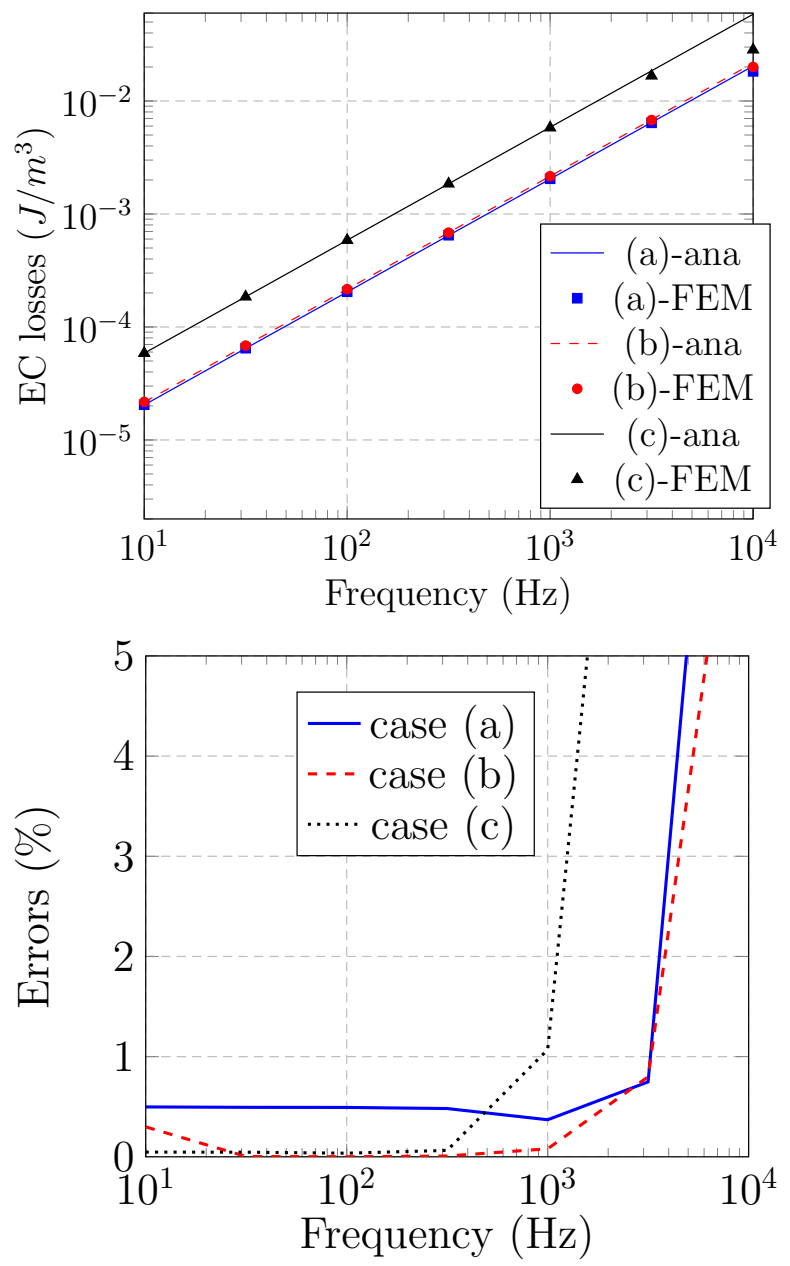

Figure 6: EC losses from homogenization model: comparison to FEM results and corresponding errors.

\section{Validity range}

In the previous part, a low frequency assumption was made, meaning that the skin depth was quite big compared to the grain size. The induced current distribution could then be approximated with simple linear functions. At higher frequency, because of skin effect, the current is restricted to a very thin layer near the conductor surfaces, as can be indicated in Fig. 7. When this assumption is not valid anymore, the current distribution can be described from Fourier series or Bessel functions [19].

From substantial simulations on homogeneous 2D geometry, we find that at frequency around $f_{s}$, which is when skin depth is $\delta=R / 2$, the EC losses reach its extreme. Thus,

$$
f_{s}=\frac{4}{\pi \mu \sigma R^{2}}
$$

where $R$ is the typical size (radius for circle and half the 

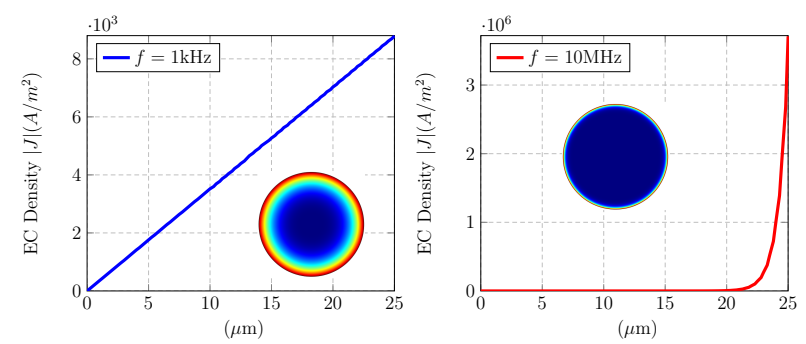

Figure 7: Distribution of electrical density of a circular from center to boundary. Material: Iron. Radius, $R=$ $25 \mu \mathrm{m}$, Magnetic induction: $10 \mathrm{mT}$.

side length for square). We find that $f<0.1 f_{s}$ provides a frequency criterion for what we describe as 'low frequency'.

\section{Conclusions}

A homogenization strategy is proposed to define the equivalent conductivity for SMC based on an estimate of EC losses. In this approach, only one analytical or numerical calculation on a heterogeneous cell and one numerical computation on a homogenized structure are needed to calculate the EC losses. The equivalent conductivity can be obtained, and then used to analytically compute EC losses for SMC with different loadings and different properties. An implementation example is given with typical SMC composition and with complex structures. The valid frequency range for this homogenization model is determined depending on conductivity, permeability and size of the ferromagnetic inclusions.

Even if real SMC is roughly made of ellipsoid inclusions, we have only considered infinitely long cylinders in this paper in order to simplify the equations involved. However, the theory and method can be similarly applied to ellipsoidal inclusions.

\section{Acknowledgement}

Xiaotao Ren is a fellowship beneficiary with the cooperation program between CSC (China Scholarship Council) and Université Paris-Sud.

\section{References}

[1] G. Cvetkovski and L. Petkovska, "Performance improvement of PM synchronous motor by using soft magnetic composite material," IEEE Trans. Magn., vol. 44, no. 11, pp. 3812-3815, Nov 2008.

[2] A. Chebak, P. Viarouge, and J. Cros, "Analytical computation of the full load magnetic losses in the soft magnetic composite stator of high-speed slotless permanent magnet machines," IEEE Trans. Magn., vol. 45, no. 3, pp. 952-955, March 2009.
[3] F. Bernot, A. Bernot, and J.-C. Vannier, Innovative Design, Analysis and Development Practices in Aerospace and Automotive Engineering: I-DAD 2014, February 22-24, 2014. New Delhi: Springer India, 2014, ch. A Synchronous Wound Excitation Transverse Flux Machine with Solid Rotor, pp. 2539.

[4] T. Sato, S. Aya, H. Igarashi, M. Suzuki, Y. Iwasaki, and K. Kawano, "Loss computation of soft magnetic composite inductors based on interpolated scalar magnetic property," IEEE Trans. Magn., vol. 51, no. 3, pp. 1-4, March 2015.

[5] H. Shokrollahi and K. Janghorban, "Soft magnetic composite materials (SMCs)," J. Mater. Process. Technol, vol. 189, no. 13, pp. 1-12, 2007.

[6] I. Niyonzima, R. Sabariego, P. Dular, and C. Geuzaine, "Finite element computational homogenization of nonlinear multiscale materials in magnetostatics," IEEE Trans. Magn., vol. 48, no. 2, pp. 587-590, Feb. 2012.

[7] O. Bottauscio and A. Manzin, "Comparison of multiscale models for eddy current computation in granular magnetic materials," J. Comp. Phys., vol. 253, pp. 1$17,2013$.

[8] A. Bossavit, "On the homogenization of Maxwell equations," COMPEL - The international journal for computation and mathematics in electrical and electronic engineering, vol. 14, no. 4, pp. 23-26, 1995.

[9] M. El Feddi, Z. Ren, A. Razek, and A. Bossavit, "Homogenization technique for Maxwell equations in periodic structures," IEEE Trans. Magn., vol. 33, no. 2, pp. 1382-1385, March 1997.

[10] G. Meunier, V. Charmoille, C. Guerin, P. Labie, and Y. Marechal, "Homogenization for periodical electromagnetic structure: Which formulation?" IEEE Trans. Magn., vol. 46, no. 8, pp. 3409-3412, August 2010.

[11] P. Queffelec, D. Bariou, and P. Gelin, "A predictive model for the permeability tensor of magnetized heterogeneous materials," IEEE Trans. Magn., vol. 41, no. 1, pp. 17-23, Jan 2005.

[12] L. Daniel and R. Corcolle, "A note on the effective magnetic permeability of polycrystals," IEEE Trans. Magn., vol. 43, no. 7, pp. 3153-3158, July 2007.

[13] C. Holloway, M. Sarto, and M. Johansson, "Analyzing carbon-fiber composite materials with equivalentlayer models," IEEE Trans. Electromagn. Compat., vol. 47, no. 4, pp. 833-844, Nov. 2005.

[14] F. Qin and C. Brosseau, "A review and analysis of microwave absorption in polymer composites filled 
with carbonaceous particles," J. Appl. Phys., vol. 111, no. 6, pp. 1-24, 2012.

[15] V. Préault, R. Corcolle, L. Daniel, and L. Pichon, "Effective permittivity of shielding composite materials for microwave frequencies," IEEE Trans. Electromagn. Compat., vol. 55, no. 6, pp. 1178-1186, Dec. 2013.

[16] A. Sihvola, "Homogenization principles and effect of mixing on dielectric behavior," Phot. Nano. Fund. Appl., vol. 11, no. 4, pp. 364-373, 2013.

[17] G. Bertotti, "Connection between microstructure and magnetic properties of soft magnetic materials," $J$. Magn. Magn. Mater, vol. 320, no. 20, pp. 2436 2442, 2008, proceedings of the 18th International Symposium on Soft Magnetic Materials.

[18] M. De Wulf, L. Anestiev, L. Dupré, L. Froyen, and J. Melkebeek, "Magnetic properties and loss separation in iron powder soft magnetic composite materials," J. Appl. Phys., vol. 91, no. 10, pp. 7845-7847, 2002.

[19] S. K. Mukerji, M. George, M. B. Ramamurthy, and K. Asaduzzaman, "Eddy currents in solid rectangular cores," Prog. Electromagn. Res. B, vol. 7, pp. 117131, 2008.

[20] O. de la Barriere, M. LoBue, and F. Mazaleyrat, "Semianalytical and analytical formulas for the classical loss in granular materials with rectangular and elliptical grain shapes," IEEE Trans. Magn., vol. 50, no. 10 , pp. 1-8, Oct 2014. 